\title{
Jones Test
}

National Cancer Institute

\section{Source}

National Cancer Institute. Jones Test. NCI Thesaurus. Code C85244.

A test for patency of the lacrimal system; fluorescein instilled in the conjunctival sac can be recovered from the inferior nasal meatus. 